\title{
Towards a Case-Based Reasoning System for Predicting Aesthetic Outcomes of Breast Reconstruction
}

\author{
Juhun LEE ${ }^{a, b}$, Clement S. SUN ${ }^{a, b}$, Gregory P. REECE ${ }^{b}$, \\ Michelle C. FINGERET ${ }^{b}$, Mia K. MARKEY ${ }^{a, b^{*}}$ \\ a The University of Texas at Austin, Austin TX, USA; \\ ${ }^{b}$ The University of Texas MD Anderson Cancer Center, Houston TX, USA \\ http://dx.doi.org/10.15221/13.279
}

\begin{abstract}
As many breast cancer survivors are candidates for multiple types of breast reconstruction, they need help visualizing possible outcomes to make optimal decisions about breast reconstruction. The purpose of this study was to develop a prototype of a system that could help women visualize possible breast reconstruction surgery results by displaying examples of reconstruction outcomes achieved by patients with similar pre-operative features. We present a prototype case-based reasoning (CBR) system that queries a database of women who have already undergone breast reconstruction surgery to retrieve a subset of cases that were pre-operatively similar to the test patient. Similarity is assessed in terms of features such as breast volume, patient age, and body mass index (BMI). In our prototype CBR system, the prior cases are reused in a straightforward manner; we simply present the post-operative $3 \mathrm{D}$ images of the cases that were pre-operatively similar to the test patient. The prototype CBR system was built on the data obtained from 47 patients. For each patient, we obtained $3 \mathrm{D}$ images before and six months after the initial breast reconstruction surgery. From these images, we quantified left and right pre-operative breast volumes. In order to retrieve cases that were pre-operatively similar to a given test patient, we applied the k-nearest neighbor algorithm (based on Euclidean distance) on the pre-operative features. We demonstrated the usefulness of our CBR system by presenting the sample query, which showed visually similar reconstructed breasts compared to the real reconstruction outcome.
\end{abstract}

Keywords: case-based reasoning, k-nearest neighbor, recommender system, breast cancer, breast reconstruction

\section{Introduction}

Many breast cancer survivors are candidates for multiple types of breast reconstruction, e.g., reconstruction using an implant (implant-based reconstruction) and/or using some of the patient's own tissue (autologous-based reconstruction). It can be difficult to decide on one option from among the alternatives. Moreover, breast cancer survivors face decisions about a series of reconstruction surgeries because a single operation is often insufficient. Thus, in order for women to make optimal decisions about breast reconstruction, they need help visualizing possible outcomes.

A patient choosing among different forms of breast reconstruction may find it beneficial to view post-operative 3D pictures of other women who have undergone the procedures that she is considering. Our hypothesis is that it would be more helpful to view postoperative 3D pictures of women who were pre-operatively similar to the patient (i.e., patients who had similar preoperative features to the test patient) rather than post-operative 3D pictures that are arbitrarily selected. In order to test this hypothesis, we need to have a system that can identify prior patients who have similar pre-operative features to the current patient, and visualize their post-operative images to her. Hence, in this study we present a prototype case-based reasoning (CBR) system that queries a database of women who have already undergone breast reconstruction surgery to retrieve a subset of cases that were pre-operatively similar to the current patient.

Similarity is assessed in terms of features such as breast volume, patient age, and body mass index (BMI). The choice of such features is based on prior studies that investigated which features are associated with differences in breast morphology. Some studies found that older age and larger BMI are correlated with higher risk of having breast ptosis [1,2]. In addition, Brown et al. [3] reported that older age is related with the inferior displacement of vertical measurements between landmarks (e.g., sternal notch to nipples), and larger weight is related with the inferolateral movement of landmarks.

*mia.markey@utexas.edu; +1-512-471-1711; bmil.bme.utexas.edu 
Moreover, the breast volume is known to be crucial information for correction of breast asymmetry in breast reconstruction [4]. In our prototype CBR system, the prior cases are reused in a straightforward manner; we simply present the post-operative 3D images of the cases that were pre-operatively similar to the current patient.

In this study, we introduced the concept of our CBR system. The system uses the k-nearest neighbor algorithm to search the patients in the database who have pre-operatively similar age, BMI, and breast volumes. In order to show the effectiveness of our CBR system, we presented sample queries and discuss the visual similarity between the post-operative images of patients returned from the system and the actual post-operative image of the queried patient.

\section{Materials and Methods}

\subsection{Dataset}

Under an IRB approved study, we obtained 3D torso images of 373 patients who had or were scheduled to undergo breast reconstruction surgery at The University of Texas MD Anderson Cancer Center between March 30, 2011 and May 17, 2013. A custom 3dMDtorso System (3dMD LLC, Atlanta GA) was used to obtain 3D torso images. Among those 373 patients, we included only patients who had 3D torso image taken at six months after the initial reconstruction. After that, the final number of patients was 47 . We extracted a total of four features from patient data: 1) age, 2) BMI, 3) left breast volume, and 4) right breast volume. The average patient age was 54 and ranged from 30 to 74 . The average BMI was 27.5 with a range of 17.9 to 40.8 . The average left breast volume was $577.8 \mathrm{cc}$ with a range of 108.11 to $1261.2 \mathrm{cc}$. The average right breast volume was slightly larger at $591.5 \mathrm{cc}$ with a range of 114.21 to $1552.51 \mathrm{cc}$. Among those, a total of 42 patients (26 underwent implant reconstruction, 15 underwent autologous reconstruction, and 1 was mixed) were used to develop our prototype CBR system and the remaining 5 patients ( 2 underwent implant reconstruction, 3 underwent autologous reconstruction) were used to test/evaluate the concept. Most patients were White $(90.1 \%)$, one was Asian (2.3\%), one was American Indian/Alaskan Native (2.3\%), one was both Asian and American Indian/Alaskan Native (2.3\%), and one was African American (2.3\%). With regard to ethnicity, most patients were non-Hispanic $(88.9 \%)$, and of those that were Hispanic, they were all White with regard to race.

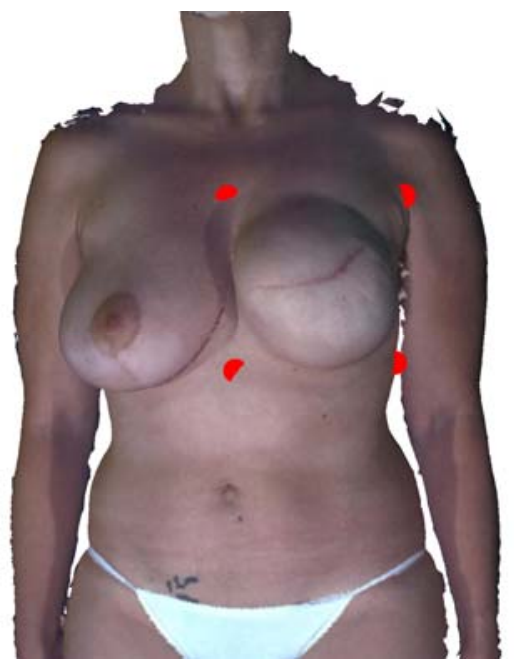

Fig 1. This figure illustrates four landmarks used for computing the volume of the reconstructed breast. Two inferior points are on the midline of the subject's torso and the other two are on the subject's anterior axillary line. The vertical locations of the landmarks were adjusted such that the Coon's Patch included the breast mound.

\subsection{Features}

Due to the limited number of patients in our database, we restricted out features space to the aforementioned four features: (1) age, (2) body mass index (BMI), (3) left breast volume, and (4) right breast volume. With an increased number of patients, more features (e.g., symmetry measures) could be included. However, these four features succinctly capture information about the patient's general physical appearance in regard to her breasts. 
Age was defined as the age of the patient at time of enrollment in our study. Each patient's BMI was obtained from the medical record. To determine volume, we used the breast volume measuring tool in $3 \mathrm{dMD}$ patient program (3dMD, Atlanta, GA) to quantify the left and right breast volume of patients. The tool requires a total of 4 points around the breast mound to compute the volume. Figure 1 illustrates the four points that we used for the volume calculation. Those four points around the breast were used to compute the Coons patch, which estimates the chest wall behind the breast. We used the anterior axillary line and the midline on the subject's torso to locate those four points. Once the Coon's patch was identified, the tool computes the volume between the surface of breast and the identified patch.

\subsection{Case-Based Reasoning System}

Our CBR system is built on the feature space spanned by the aforementioned four features obtained from patients at pre-operative stage. Each pre-operative data is linked with the same patient's data at 6 months post-operative stage. If the data of one patient at pre-operative stage are entered, the system searches for similar cases based on the pre-operative data; then, their corresponding post-operative data at 6 months are retrieved. In order to search for cases that were pre-operatively similar to a given test patient, we applied the k-nearest neighbor algorithm (based on Euclidean distance) on the pre-operative data space. From their post-operative images, breast cancer survivors would be able to check the breast reconstruction results of patients who had pre-operatively similar BMI, age and breast volumes. Figure 2 depicts the overview of our CBR system.

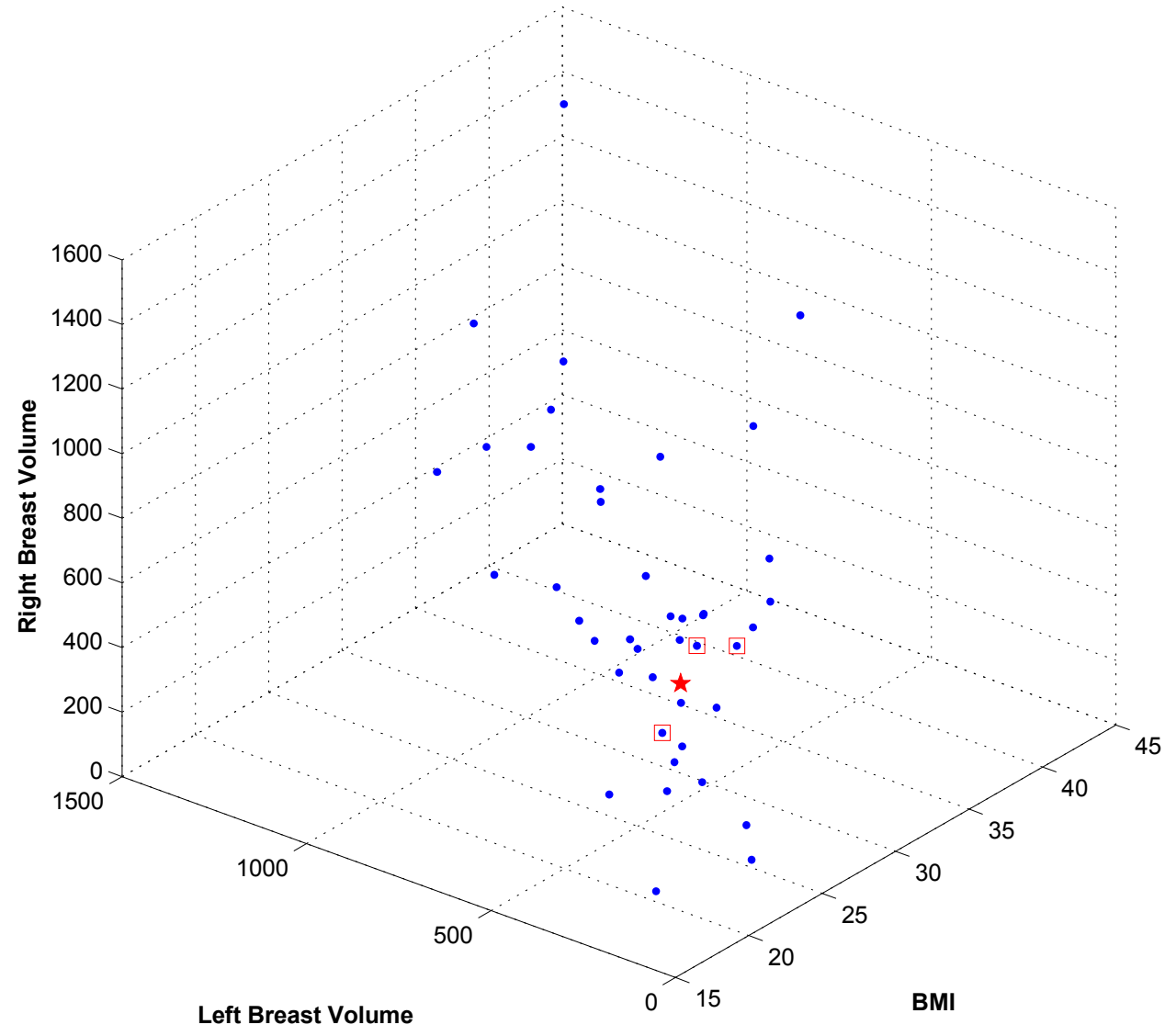

Fig. 2. Diagram showing the concept of our CBR system. One dimension (Age) was omitted for visualization purposes. Once a patient's data (star) are entered, the k-nearest algorithm searches for the $k$ most similar examples in the data space, which was spanned by the existing patients' data. In this figure, $k$ was selected as 3.

\section{Results}

Table 1 shows the demographic detail (age, BMI, left and right breast volume, ethnicity, race, and her final choice of reconstruction method) of patients in the test set and their corresponding $k$-nearest neighbors. For the $k$-nearest neighbor algorithm, we set $k=3$. Figure 3 shows that the possible results (two among the three nearest neighbors) obtained from our CBR system were visually similar to the 
actual results for one test patient (ID \# T4). The 6-month result of the first nearest neighbor is the patient who underwent bilateral reconstruction with tissue expanders (ID \# K8). The 6-month result of the second nearest neighbor is the patient who underwent unilateral reconstruction with a tissue expander (ID \# K9). In fact, the selected test patient underwent an immediate bilateral transverse rectus abdominis reconstruction. Although her ultimate choice was different from those two selected examples, the first nearest neighbor result (ID \# K8) is visually very similar to her choice.

Table 1. Profiles of the five test patients and their k-nearest neighbors.

\begin{tabular}{|c|c|c|c|c|c|c|c|c|}
\hline $\begin{array}{c}\text { Test } \\
\text { Patients }\end{array}$ & $\begin{array}{c}\text { k-nearest } \\
\text { neighbors } \\
(k=3)\end{array}$ & Age & BMI & $\begin{array}{c}\text { Left } \\
\text { Breast } \\
\text { Volume } \\
\text { (cc) }\end{array}$ & $\begin{array}{l}\text { Right } \\
\text { Breast } \\
\text { Volume } \\
\text { (cc) }\end{array}$ & Ethnicity & Race & $\begin{array}{l}\text { Reconstruction } \\
\text { Method }\end{array}$ \\
\hline \multirow[t]{4}{*}{ T1 } & & 47 & 22.3 & 276.55 & 605.43 & $\begin{array}{c}\text { Not } \\
\text { Hispanic }\end{array}$ & White & Autologous \\
\hline & K1 & 59 & 25.9 & 375.56 & 587.61 & $\begin{array}{c}\text { Not } \\
\text { Hispanic }\end{array}$ & White & Implant \\
\hline & $\mathrm{K} 2$ & 57 & 28.8 & 382.59 & 508.32 & $\begin{array}{c}\text { Not } \\
\text { Hispanic }\end{array}$ & White & Implant \\
\hline & K3 & 73 & 22.4 & 330.3 & 427.6 & $\begin{array}{c}\text { Not } \\
\text { Hispanic }\end{array}$ & White & Implant \\
\hline \multirow[t]{4}{*}{ T2 } & & 35 & 24 & 324.58 & 496.92 & $\begin{array}{c}\text { Not } \\
\text { Hispanic }\end{array}$ & White & Implant \\
\hline & K2 & 57 & 28.8 & 382.59 & 508.32 & $\begin{array}{c}\text { Not } \\
\text { Hispanic }\end{array}$ & White & Implant \\
\hline & K3 & 73 & 22.4 & 330.3 & 427.6 & $\begin{array}{c}\text { Not } \\
\text { Hispanic }\end{array}$ & White & Implant \\
\hline & K4 & 34 & 24.9 & 378.77 & 435.68 & Hispanic & White & Implant \\
\hline \multirow[t]{4}{*}{ T3 } & & 59 & 27.1 & 280.5 & 337.51 & $\begin{array}{c}\text { Not } \\
\text { Hispanic }\end{array}$ & White & Implant \\
\hline & K5 & 42 & 21.5 & 281.4 & 291.54 & $\begin{array}{c}\text { Not } \\
\text { Hispanic }\end{array}$ & Asian & Autologous \\
\hline & K6 & 52 & 18.8 & 330.71 & 330.69 & $\begin{array}{c}\text { Not } \\
\text { Hispanic }\end{array}$ & White & Implant \\
\hline & K7 & 66 & 22.7 & 233.96 & 307.39 & $\begin{array}{c}\text { Not } \\
\text { Hispanic }\end{array}$ & White & Implant \\
\hline \multirow[t]{4}{*}{ T4 } & & 55 & 30 & 765.59 & 739.84 & $\begin{array}{c}\text { Not } \\
\text { Hispanic }\end{array}$ & White & Autologous \\
\hline & K8 & 53 & 22.6 & 793.51 & 719.51 & $\begin{array}{c}\text { Not } \\
\text { Hispanic }\end{array}$ & White & Implant \\
\hline & K9 & 46 & 28.9 & 755.86 & 796.65 & $\begin{array}{c}\text { Not } \\
\text { Hispanic }\end{array}$ & White & Implant \\
\hline & K10 & 60 & 30.3 & 812.77 & 776.59 & Hispanic & White & Autologous \\
\hline \multirow[t]{4}{*}{ T5 } & & 59 & 38 & 763.12 & 752.35 & $\begin{array}{c}\text { Not } \\
\text { Hispanic }\end{array}$ & White & Autologous \\
\hline & K9 & 46 & 28.9 & 755.86 & 796.65 & $\begin{array}{c}\text { Not } \\
\text { Hispanic }\end{array}$ & White & Implant \\
\hline & K8 & 53 & 22.6 & 793.51 & 719.51 & $\begin{array}{c}\text { Not } \\
\text { Hispanic }\end{array}$ & White & Implant \\
\hline & K10 & 60 & 30.3 & 812.77 & 776.59 & Hispanic & White & Autologous \\
\hline
\end{tabular}




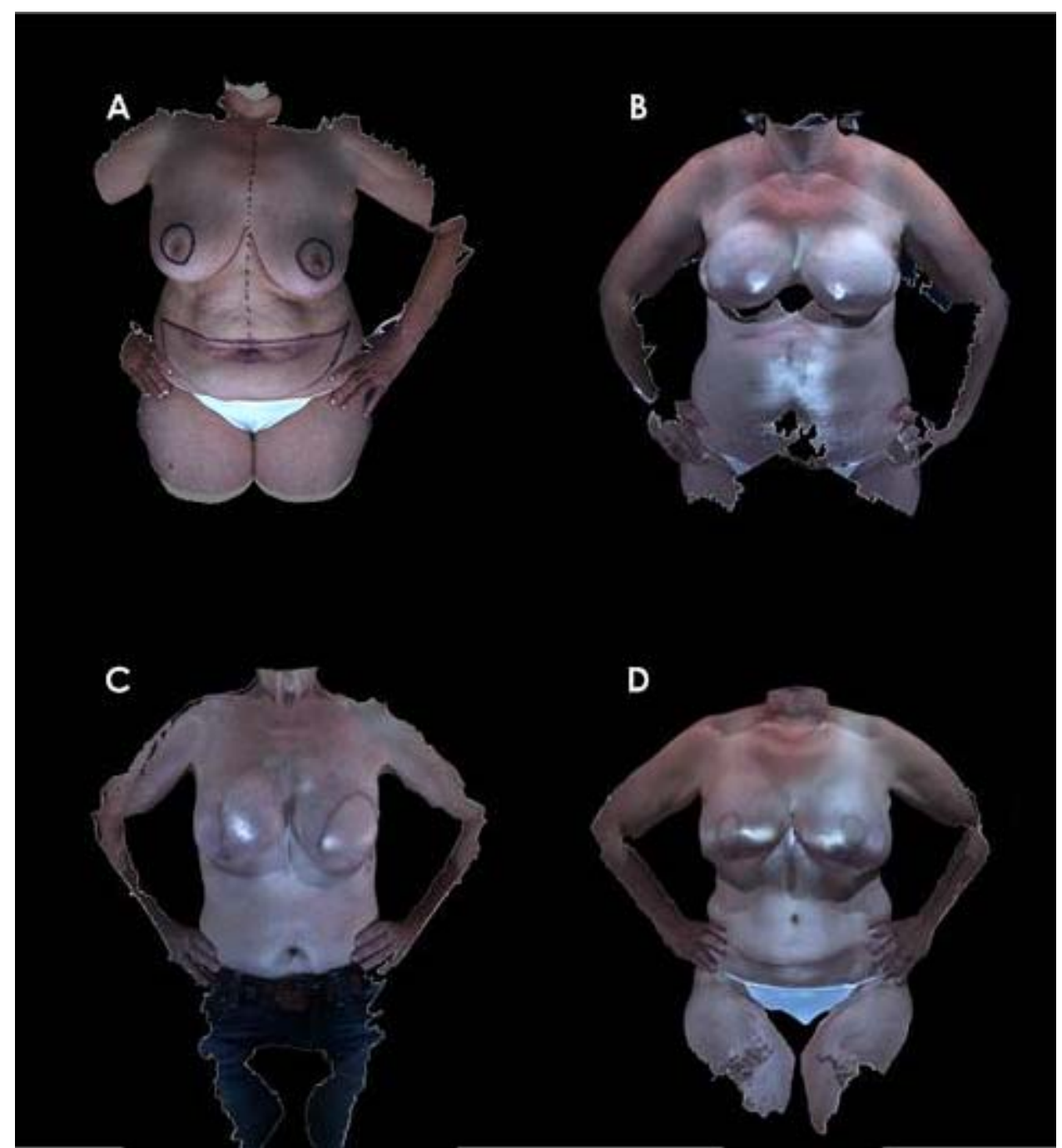

Fig. 3. An example result from our CBR system. (A) This figure depicts the pre-operative image of one test patient (ID \# T4). She was 55 years old with a BMI of 30. Suppose her pre-operative information (her breast volumes, BMI, and age) is entered to our system. Then, it searches the three nearest neighbors (i.e., pre-operative patients who have similar age, BMI, and breast volumes) from the dataset. From their post-operative images, she would be able

to check the breast reconstruction results of patients who had similar BMI, age and breast volumes. As an example, we show two 6-month results (depicts in $B$ and $C$ ) among the three nearest neighbors that she would be

able to check from our system. (B) This figure shows the first nearest neighbor who underwent bilateral reconstruction with tissue expanders. (C) This figure shows the second nearest neighbor who underwent unilateral reconstruction with a tissue expander. In fact, the test patient underwent an immediate bilateral transverse rectus abdominis reconstruction with her actual 6-month result shown in (D). Although her ultimate choice was different from those two selected examples, the first nearest neighbor result is visually very similar to her ultimate choice.

\section{Discussions and Conclusion}

In this study, we presented a prototype of a case-based reasoning system that may help a breast cancer survivor decide among her breast reconstruction options by presenting post-operative 3D images of cases that were pre-operatively similar to her. The system searches the database for patients with preoperative features similar to those of the test patient but who already underwent breast reconstruction. Age, BMI, and breast volumes were used as features to assess the similarity. The $k$-nearest neighbor algorithm was used to search for the pre-operative similar cases. We showed the effectiveness of our system by presenting visually similar post-operative outcomes comparing to the actual outcome of the queried patient.

As briefly mentioned, we restricted out features space to age, BMI, and breast volumes due to the limited sample size of our database. With a larger reference database, other features that are closely related with breast morphology can be added to enrich the feature space, and therefore improve the 
system. Two types of features can be included for the system: 1) quantitative measures of breast morphology such as Breast Retraction Assessment (BRA) [5], breast ptosis measures [6,7], and the curvature of breast contour [8] and 2) patient history data that can affect breast morphology such as the number of children, number of years breast feeding, and history of weight loss [2].

The future study includes 1) validating the system with a larger dataset, 2) incorporating the above possible additional features, and 3 ) evaluating the effectiveness of the system on other race/ethnic groups (non-Hispanic white is the dominant race/ethnic group of our dataset).

\section{References}

1. B. Rinker, M. Veneracion, and C. P. Walsh, (2008): "The Effect of Breastfeeding on Breast Aesthetics," Aesthet. Surg. J., vol. 28, no. 5, pp. 534-537.

2. B. Rinker, M. Veneracion, and C. P. Walsh, (2010): "Breast ptosis: causes and cure," Ann. Plast. Surg., vol. 64, no. 5, pp. 579-584.

3. T. P. L. H. Brown, C. Ringrose, R. E. Hyland, A. A. Cole, and T. M. Brotherston, (1999): "A method of assessing female breast morphometry and its clinical application," Br. J. Plast. Surg., vol. 52, no. 5, pp. 355-359.

4. M. S. Kim, J. C. Sbalchiero, G. P. Reece, M. J. Miller, E. K. Beahm, and M. K. Markey, (2008): "Assessment of breast aesthetics," Plast. Reconstr. Surg., vol. 121, no. 4, p. 186e-94e.

5. R. D. Pezner, M. P. Patterson, L. R. Hill, N. Vora, K. R. Desai, J. O. Archambeau, and J. A. Lipsett, (1985): "Breast retraction assessment: an objective evaluation of cosmetic results of patients treated conservatively for breast cancer," Int. J. Radiat. Oncol. Biol. Phys., vol. 11, no. 3, pp. 575-578.

6. P. Regnault, (1976): "Breast ptosis. Definition and treatment," Clin. Plast. Surg., vol. 3, no. 2, pp. 193-203.

7. M. S. Kim, G. P. Reece, E. K. Beahm, M. J. Miller, E. N. Atkinson, and M. K. Markey, (2007): "Objective assessment of aesthetic outcomes of breast cancer treatment: measuring ptosis from clinical photographs," Comput. Biol. Med., vol. 37, no. 1, pp. 49-59.

8. J. Lee, S. Chen, G. P. Reece, M. A. Crosby, E. K. Beahm, and M. K. Markey, (2012): "A Novel Quantitative Measure of Breast Curvature Based on Catenary," IEEE Trans. Biomed. Eng., vol. 59, no. 4 , pp. $1115-1124$. 